\title{
Knowledge, Source of Knowledge, and Perceived Barriers of Family Planning Among Women Attending Reproductive Health Clinics in Suva, 2017
}

\author{
Jay L ${ }^{1}$, Masoud $\mathbf{M}^{2 *}$, Sabiha $\mathbf{K h}^{3}$ and Avelina $\mathbf{R}^{4}$ \\ ${ }^{1}$ MPH student, Fiji National University, Fiji \\ ${ }^{2}$ Associate Professor, Fiji National University, Fiji \\ ${ }^{3}$ Lecturer, Fiji National University, Fiji \\ ${ }^{4}$ Coordinator, Pacific Sexual \& Reproductive Health Research Centre, Fiji National \\ University, Fiji
}

*Corresponding author: Masoud Mohammadnezhad, Associate Professor in Public health (Health promotion), School of Public Health and Primary Care, Fiji National University, Fiji, Email: masoud.m@hotmail.com

\section{Abstract}

Introduction: The Global Total Fertility (GTF) rate is projected to decline to 2.4 children per woman by 2030 and 2.2 by 2050. The importance of family planning in promoting maternal and child health is undeniable. Due to lack of previous study, this study aims to identify the level of knowledge, source of knowledge, and perceived barriers of family planning among women of reproductive age in Suva, Fiji.

Method: This is a quantitative, cross-sectional study which used a self-administered questionnaire among 325 participants who attended three designated health clinics around Suva, Fiji from 15 March to 28 April 2017. Participants who met the inclusion and exclusion criteria who consented to participate in the study were asked to fill a questionnaire survey. Once the data was collected, it was analyzed with SPSS. P value less than $0.05 \%$ was considered as the level of significance.

Results: The mean age of the participants was 31.93 \pm 7.35. A majority of the participants were i Taukei women (65.2\%) and $60.9 \%$ held a post-secondary school degree. Overall, participants had poor knowledge about the mechanism of action of contraceptives. $74.5 \%$ of the participants reported the side effects as the main reason for not using contraceptives. Most of the participants (82.2\%) received their information about family planning from heath care providers.

Conclusion: The importance of this study is evidence that it is one of a very few studies concerned with the state of knowledge about family planning in Fiji and as such the study provides a useful source of empirical information to policy makers to achieve the desired goals in family planning. These findings of the study will help health care providers promote family planning in Fiji.

Keywords: Family Planning; Knowledge; Barriers; Reproductive Health; Fiji 


\section{Introduction}

Family planning is the practice of controlling the number of children and the spacing between children. The World Health Organization (WHO) defines family planning as the act(s) that allow individuals and couples to anticipate and attain the desired number, timing, and spacing of their children [1]. Family planning eliminates poverty, reduces the rate of deaths of infants, encourages development of economy, and gives power to women [2]. Other benefits include ensuring women's empowerment to make decisions on their family size, reduction of maternal and neonatal mortalities, prevention of sexually transmitted infections (STIs), reduction of pregnancyrelated health risks for women, reduction of unsafe abortion and last but not least, reduction of adolescent pregnancies. Family planning is not only cost effective but it is beneficial in terms of social, economic, health and environmental benefits [3]. Despite the benefits of family planning, there are approximately 220 million throughout the world who have an unmet need for modern contraception and the number of likely to increase in the near future due to the increasing population [4].

Globally, the fertility patterns have declined considerably to low levels; however, there is still an increasing population trend. Sub-Saharan Africa has the highest fertility at 4.7 children per woman. The Total Fertility Rate (TFR) globally is 2.5 children per woman. Most of Europe and developed Asian countries, on the other hand, have the lowest fertility at 1.6 and 2.2 children per woman respectively. The Global Total Fertility rate is projected to decline to 2.4 children per woman by 2030 and 2.2 by 2050 [5]. Oceania or Pacific Island Countries (PICs) has a declining TFR of 2.4 in the last four decades although the TFR still remains high for most Pacific Island Countries (PICs). Fiji has a TFR of 2.6 as of 2015 which is projected to decline to 2.3 children per woman by 2030 . In terms of family planning, the PICs Contraceptive Prevalence Rate (CPR) remain well below the developing country average of 62 percent which means that a significant portion of women in the reproductive age group (15-49) who want to space and plan their number of children are not on any contraception [6]. Despite the slight improvement of contraceptive prevalence rate (CPR) from 41 percent in 1971 to 44 percent in 2012 in Fiji, there exists a knowledge gap regarding contraception [7]. Although generally a majority of women of reproductive age in Fiji may be aware of modern contraceptives, the utilization of family planning has not improved in the past four decades which means there are still unresolved barriers to family planning.
The importance of this study is evidenced by the fact that it is one of very few studies concerned with the state of knowledge of family planning in Fiji and as such it provides a useful source of empirical information. This study aims to identify the level of knowledge, source of knowledge, and perceived barriers of family planning among women attending reproductive health clinics in Suva, Fiji in 2017. Gaining a better understanding of the perceived barriers and source of knowledge of family planning are valuable for developing service promotion strategies and service delivery protocols by health care policy makers.

\section{Methods}

This quantitative, cross-sectional study was conducted among 325 participants who attended three designated health clinics around Suva, Fiji from 15 March to 28 April 2017. A self -administered questionnaire was used to collect data, which was authenticated and face validated by three professionals in related expertise to ensure the substance was suitable. The three randomly selected family planning health clinics were Nuffield Clinic in Tamavua, Wellness Center for Women/Oxfam) at the Colonial War Memorial Hospital (CWMH) in Suva, and Samabula Health Center in Suva. The study population included all Fijian women of reproductive age. The inclusion criteria included women, ages 15-49 that were married and must have attended any one of the three identified family planning clinics under study from 15 March to 28 April 2017. Furthermore, the study participant must have been a Fijian-anybody in Fiji who possesses Fijian citizenship. The exclusion criteria included men and women under the age of 15 or age 50 and over and those women who are not happy to participate in the study. Participants who met the inclusion and exclusion criteria who consented to participate in the study were asked to fill a questionnaire survey to identify the level of knowledge, source of knowledge on family planning, and the barriers to their practice of family planning. Data collection was done after the researcher received ethical approval from the Ethics Review Committee (FNREC) and College of Health Research Ethics Committee (CHREC) and also MHMS Fiji National Heath Research and. The data was analyzed with SPSS Version 22 statistical software. Data analyzed considered a $p$ value of $0.05 \%$ and a $95 \%$ confidence interval.

\section{Results}

The sample was comprised of 325 women ages 15-49 $(\mathrm{M}=31.93 \pm 7.35)$. With respect to age, participation was 
highest, $83(25.54 \%)$ in the age range of $26-30$ while the lowest was the age group 15-19, 8 (2.08\%). In terms of ethnicity, the majority of the sample, 212 (62.2\%) stated that they were native Fijians or that they belonged to iTaukei ethnic group while the smallest number of participants was Chinese at $2(0.6 \%)$. All of the participants in the study were married. A majority of the women $(60.9 \%)$ had a higher education degree. Only 14 $(0.3 \%)$ of the women in the sample had an education at the primary level. In terms of religious affiliation, a vast majority of the participants considered themselves to be a Christian, 234 (72.1\%). A small fraction of the participants were in other minority religious groups, 22 (7.7\%) (Table 1).

\begin{tabular}{|c|c|c|}
\hline Variable & Frequency & Percentage \\
\hline Age & & \\
\hline $15-19$ & 5 & 1.54 \\
\hline $20-24$ & 48 & 14.77 \\
\hline $25-29$ & 87 & 26.77 \\
\hline $30-34$ & 72 & 23.08 \\
\hline $35-39$ & 55 & 22.15 \\
\hline $40-44$ & 37 & 16.92 \\
\hline $45-49$ & 21 & 6.46 \\
\hline Ethnicity & & \\
\hline I-Taukei & 212 & 62.2 \\
\hline Indian & 88 & 27.1 \\
\hline Chinese & 2 & 0.6 \\
\hline Other & 23 & 7.1 \\
\hline Education & & \\
\hline Primary & 14 & 4.3 \\
\hline Secondary & 113 & 34.8 \\
\hline Higher & 198 & 60.9 \\
\hline Religion & & \\
\hline Christians & 234 & 72.1 \\
\hline Hindu & 69 & 21.2 \\
\hline Others & 22 & 7.7 \\
\hline
\end{tabular}

Table 1: Characteristics of participants $(\mathrm{N}=325)$

Overall, participants had poor knowledge about the mechanism of action of contraceptives. About a sixth of the participants incorrectly answered that birth control pills would still be effective even if a woman missed taking them for two or three days in a row, 191 (58.8\%). Almost three quarters of the participants incorrectly answered that there was an increased risk of breast cancer in women who take estrogen-containing contraceptives, 74 (22.8\%). Likewise, 143 (44.0\%) of the participants incorrectly answered that using the pill increases a woman's risk of ovarian, endometrial or cervical cancer (Table 2).

\begin{tabular}{|c|c|c|}
\hline Questions & Frequency & Percentage \\
\hline $\begin{array}{c}\text { Birth control pills are effective } \\
\text { even if a woman misses taking } \\
\text { them for two or three days in a } \\
\text { row }\end{array}$ & 191 & 58.8 \\
\hline $\begin{array}{c}\text { There is an increased risk of } \\
\text { breast cancer in women taking } \\
\text { estrogen-containing } \\
\text { contraceptives }\end{array}$ & 74 & 22.8 \\
\hline $\begin{array}{c}\text { Using the pill increases a } \\
\text { woman's risk of ovarian, } \\
\text { endometrial or cervical cancer }\end{array}$ & 143 & 44.0 \\
\hline
\end{tabular}

Table 2: Frequency of correct answers to knowledge of the mechanism of the action of contraceptives.

As shown in Figure 1, the most common reason women choose not to use contraceptives was side effects of contraceptives, 242 (74.5\%). Religion was the second most common reason, 222 (68.3\%), followed by disapproval from partner or husband, 164 (50.5\%), cultural beliefs, 132 (40.6\%). Failure and discomfort came in fifth and sixth place respectively, 117 (36\%) and 95 $(29.3 \%)$.

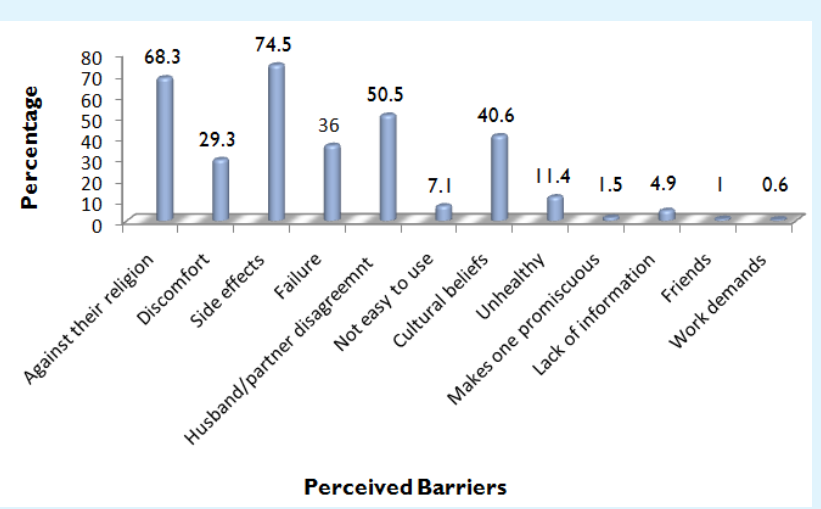

Figure 1: Distribution of participants' response based perceived barriers.

Figure 2 below shows the distribution of the source of knowledge about family planning. Most of the participants in this study received their information about family planning from heath care providers through health education, 267 (82.2\%). 103 (31.7\%) got their knowledge on family planning through friends. The mass media was another major source of knowledge on family planning, $87(26.8 \%)$. A small fraction of the participants received 
their knowledge about family planning from their husbands, $13(4 \%)$. A very tiny fraction of the participants, $6(2 \%)$ received their knowledge about family planning from their sisters and mothers.

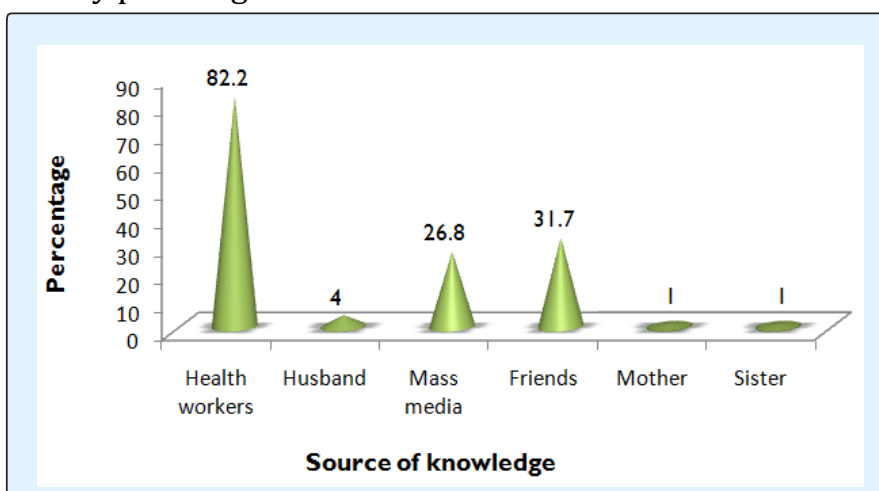

Figure 2: Distribution of participants' response based on the sources of knowledge.

\section{Discussion}

The results of this study showed that women had very poor knowledge about the mechanism of action of contraceptives. The gap between knowledge about family planning and the actual use of that knowledge has been observed in the literature. Nansseu, et al. made the same observation in the context of Zambia while Mahadeen, et al. noted that in Jordan, a large number of women had quite positive attitudes towards family planning but they simply lacked the relevant knowledge and their practices were quite bad as well $[8,9]$. When it comes to explaining the existence of this gap between knowledge about family planning, on the one hand, and attitudes and practices on the other, the notion of cultural factors seems to play a crucial role. For example, Pegu (2014) showed that the culturally conditioned desire for large families and the husband's objection to the use of contraceptives were among the main reasons why women who knew a lot about family planning simply did not practice family planning methods at a satisfactory level [10]. Therefore, it can be reasonably concluded that perceived barriers to family planning such as culture and religion, among others, can place barriers to a woman's ability to acquire knowledge on family planning.

When it comes to the source of knowledge of family planning, health education was the obvious choice of the majority of the women, followed by friends, mass media, husband and others. As expected, health education should be the primary source of knowledge in disseminating information on family planning to women. The source of information is essential for women in reproductive age as they realize reproductive rights are part of their human rights. The major source of information regarding family planning is received from health care facilities during the education forums that are offered as part of antenatal programs. Health personnel educate people on the importance of contraceptives while, friends, media, family members, and relatives are informative on different contraceptives, their side effects and benefits. Therefore, the knowledge and skills of health personnel is essential in continuously enforcing and enhancing sound contraceptives advice [11]. Lastly, the source of information enables reproductive women to make sound choices of contraceptive, avoid reliance of a single method, and send insight on contraceptive methods.The content of information that women receive is important in enhancing their understanding of family planning and initiation of usage.Information ensures that the women who use family planning do it in the right manner as well as trigger the ones who do not use to start. Indeed, the information is useful in training women on the availability of contraceptives, their benefits, related side effects, and the importance of spousal consent. It is important to deal with the challenges of information gap, poor data management, low information utilization, and data inconsistencies for effective and efficient knowledge of family planning as noted by La Vincente, et al. [12].

The commonest perceived barriers to family planning from these women were side effects, religion, husband or partner's objection, culture, failure and discomfort in that particular order. Myths and misconceptions play a critical role in use of contraceptives by spreading negative attitude across communities. Myths in Fiji regarding family planning are a hindrance to family planning knowledge as stated by Naz [13]. They include the belief that use of some contraceptives has unpleasant side effects such as cancer, vomiting, irritability, early menopause, baby malfunction, bleeding disorders, weight gain, and infertility. Misconceptions regarding contraceptives make some women to avoid birth control mechanisms. They include the idea that family planning has devastating side effects that are harmful to an individual's health; [11] this results in low use of some contraceptives, provider bias, limited choices, and inadequate counseling and information. They also believe that contraceptives are less effective and they are deemed to fail. The culture in Fiji is also important in promoting use of contraceptives by encouraging women to make informed decisions that affect their lives positively by granting them an opportunity for education and better employment. Additionally, myths in Fiji, especially about the negative side effects of family planning, plays an important role in prohibiting women from controlling birth or leads to over-reliance of a single method as 
explained by Kaitani [14]. According to Kaitani (2012), male participation in family planning also contributes to knowledge, attitudes, and birth control practices. Some reproductive women hold back from using contraceptives due to lack of support from their spouses or end up making less informed decisions.In reference to the above factors, family planning services are unavailable to some Fijian women despite their being critical due to financial challenges, lack of awareness, logistical barriers, and cultural background [15].

\section{Conclusion}

These findings painted a picture of the participants as women in their prime to give birth. A sample of knowledge questions revealed that among these participants there was poor knowledge on family planning. The study identified side effects, religious beliefs, husband objection, cultural beliefs, failure of contraceptives and discomfort as the most common barriers to family planning. Over half of the participants received their information about family planning through health education, followed by friends, mass media and lastly by the husband and others.

Deriving policy recommendations from empirical results is one of the main reasons why such studies are worthwhile. Given that the results of the present study have not produced any definitive conclusions, some policy recommendations can be derived from the tentative conclusion about the inverse distribution family planningrelated knowledge and practices across socio-economic strata.

\section{References}

1. WHO (2016) Family planning.

2. Tizta T, Gily C, Stanley L, Wondwosen K, Els L, et al. (2013) Family planning knowledge, attitude and practice among married couples in Jimma zone, Ethiopia. Ethiopia Journal of Health Dev 19: 37-44.

3. World Health Organization (2015b) Family Planning/Contraception. Family planning fact sheet.

4. Cates W, Stanback J, Maggwa B (2014) Global family planning metrics-time for new definitions? Contraception 90(5): 472-475.

5. World Fertility Patterns (2015) United Nations Economic and Social Affairs.
6. Population \& Developmental Profiles: Pacific Island Countries (2014) United Nations Populations Fund pp. 2-6.

7. Ministry of Health (2013) Tracking Progress in Maternal and Child Survival. A Case Study Report pp. 7-17.

8. Nansseu JR, Nchinda EC, Katte JC, Nchagnouot FM, Nguetsa GD (2015) Assessing the knowledge, attitude and practice of family planning among women living in the Mbouda health district. Reproductive Health 12(92).

9. Mahadeen AI, Khalil AO, Hamdan-Mansour AM, Sato T, Imoto A (2012) Knowledge, attitudes and practices towards family planning among women in the rural southern region of Jordan. East Mediterr Health J 18(6): 567-571.

10. Pegu B, Gaur BPS, Sharma N, Singh AS (2014) Knowledge, attitude and practices of contraception among married women. International Journal of Reproduction, Contraception, Obstetrics, and Gynecology 3(2), 385-388.

11. Fried S, Harrison B, Starcevich K, Whitaker C, OKonek $T$ (2012) Integrating interventions on maternal mortality and morbidity and HIV: A human rights based framework and approach. Health Hum Rights, 14(2): 21-33.

12. La Vincente SF, Mielnik D, Jenkins K, Bingwor F, Volavola L, et al. (2015) Implementation of a national school-based Human Papillomavirus (HPV) vaccine campaign in Fiji: Knowledge, vaccine acceptability and information needs of parents. BMC Public Health, 15(1): 1257.

13. Naz R (2014) Sex education in Fiji. Sexuality \& Culture 18(3): 664-687.

14. Kaitani MM (2012) Bridging the Gap: the Changing Reproductive and Sexual expectations of Fijian men. Open Access Theses.

15. Delaibatiki R (2016) Knowledge, Attitudes and Practice of family planning among iTaukei women in Fiji and New Zealand (Thesis, Doctor of Philosophy). University of Otago. 